\title{
Improving Ceramic Processing Through the Application of Microstructure Mining
}

\author{
Ian Nettleship
}

Received: 10 May 2013/Revised: 19 September 2013/Accepted: 7 October 2013/Published online: 18 October 2013

(C) Springer Science+Business Media New York and ASM International 2013

\begin{abstract}
Microstructure mining aims to study material microstructures while embracing their complexity. The general steps in the microstructure mining method will be introduced in the context of ceramics processing and microstructure evolution in sintering. In particular, the methodology will be illustrated by the design of a new microstructural measurement called the effective diffusion distance. This parameter allows the experimental study of sintering microstructures using traditional kinetic models of sintering even when the microstructures are strongly aggregated. The limits of this approach will be discussed with respect to the scale of the aggregation relative to the size of the pores.
\end{abstract}

Keywords Microstructure mining - Ceramics processing $\cdot$ Sintering

\section{Introduction}

Materials informatics has been suggested as a means of accelerating materials innovation [1]. This has been reinforced by the proposed sub-discipline of Integrated Computational Materials Engineering that will require searchable databases to allow the power of computational materials engineering to be utilized to its full potential [2].

This article is an invited paper selected from presentations at the 2013 Quantitative Metallography Conference and Exposition, held April 4-5, 2013, in San Antonio, Texas, and has been expanded from the original presentation.

I. Nettleship $(\bowtie)$

Department of Mechanical Engineering and Materials Science,

University of Pittsburgh, Pittsburgh, PA 15261, USA

e-mail: nettles@pitt.edu
In the US, this concept has recently become central to a broad approach called the Materials Genome Initiative for Global Competitiveness [3]. The latter aims to develop the resources and training necessary for integrating computational and experimental materials science and engineering in a bid to increase the rate at which new materials are discovered and developed. Three enabling aspects of the required "materials innovation infrastructure" have been identified. They include: (i) computational tools, (ii) experimental tools, and (iii) digital data. Digital data can be the result of computational or experimental studies conducted by members of the materials research community which must be assembled into data sharing systems that facilitate multidisciplinary materials development. Familiar databases for thermodynamic and crystallographic data already exist, but as yet this approach has not been widely applied at the microstructure level and there are no comprehensive microstructural databases that can accept and preserve microstructural information generated by the materials community. To establish this form of database there must be agreement on the nature of the information that should be stored, the standards that must be met and the organizations that will maintain and manage the databases. Obviously this constitutes a tremendous undertaking but if it were to be successful, material informatics methods could be widely applied to microstructural information obtained by imaging. Microstructure mining, the subject of this paper, is a conceptual methodology by which quantitative statistical microstructure information can be acquired, assembled into databases, and analyzed using available data mining techniques [4]. At the heart of this method is the concept that materials microstructures can be treated as complex systems in which material properties and behavior are controlled by clearly identifiable lengthscales such as grain size or by microstructural 
"mesoscales" associated with groups of features. Quantifying lengthscales associated with individual and readily identifiable features is usually straightforward but quantification of mesoscales created by the spatial arrangements of features can be much more challenging. Previous studies have shown how microstructure mining can be used to conduct process modeling at the microstructural level in the study powder processing of ceramics and its effect on fracture strength $[5,6]$.

In this paper, the application of microstructure mining to the study of sintering kinetics will be reviewed. In particular, the effect of aggregation on the sensitivity of sintering kinetics to microstructure will be demonstrated. The traditional microstructural parameters used in kinetic sintering models are limited to the solid volume fraction and the average grain size, the latter being taken to represent the controlling diffusion distance [7, 8]. In consequence, there is an implicit assumption that the representative volume element in a sintering microstructure contains only one grain and its attendant porosity. While this is a wellaccepted simplification, there has been few attempts to validate the assumption and microstructural measurements do not play a central role in quantification of sintering kinetics by methods such as the master sintering curve [9]. The specific assumptions concerning the simplified microstructural geometries and how they evolve in sintering lead to fixed relationships between solid volume fraction and grain size. DeHoff termed the relationship between two microstructural parameters during microstructural evolution as a microstructural pathway [10]. In the study of sintering, the relationships between solid volume fraction and grain size are called microstructure maps and have been used to examine the effect of processing variables on sintering and grain growth [11-13]. Since the sintering models are applied independent of scale, dimensionless microstructural parameters have been used to compare the models with experimental data [14]. It was found that the simplifying assumptions of the models often misrepresent real microstructure evolution and the pores in the models are too often too small and too numerous. This problem becomes worse when the models are compared with microstructural evolution in ceramics derived from aggregated chemically prepared nanocrystalline powders. The general steps in the microstructure mining process will now be described and then an example of its application to the sintering of aggregated powders will be presented.

\section{Microstructure Mining Methodology}

Materials scientists are well aware that the properties of engineering material must be controlled over a range of lengthscales because of the presence of several different microstructural features with widely differing sizes. Additionally, material properties such as fracture strength may also be controlled by associations of features such as aggregations that define "mesoscales" in the microstructure and introduce additional complexity. Therefore, the potential for microstructural complexity must be considered in decisions made at every stage of microstructural analysis from sampling of the original specimens to design of the image processing and analysis protocols. Decision support is the main focus of the microstructure mining method. In the first step, the material must be divided to make smaller samples suitable for imaging. This must involve careful sampling and specimen preparation. The second step involves sampling the prepared specimen using a qualitative imaging exercise in the microstructure mining process that surveys the specimen over a wide range of magnifications to establish a "microstructural profile" that would further support decisions in the next step of the process. The next step involves choosing a magnification for the subsequent analysis. This will be relatively straightforward if the property in question is controlled by the lengthscale of a single identifiable feature but much less so when mesoscales are formed by associations of features. The spatial arrangement of features in such associations can result in much larger lengthscales and this is indeed the case in the following example that concerns the sintering of aggregated nanocrystalline ceramic powders. Once the appropriate lengthscale has been identified decisions must be made about image sampling and imaging conditions. Obviously, microstructure mining will not result in databases that are comprehensive enough to truly address all structure-property relationships for a specific material. Once the imaging is completed the images must be banked and processed. Sometimes hundreds of images may be necessary and so automated image processing protocols must be designed and implemented. This often requires compromises that must be fully identified and evaluated. In the final step, the data is extracted from the processed images and can be analyzed using data mining or pattern recognition techniques if necessary. Finally, the processed images can also be banked. Figure 1 illustrates the general steps in microstructure mining that are used to support decisions about microstructure imaging and analysis.

\section{Example: A Microstructure Mining Tool for Studying the Effect of Aggregation on the Kinetics of Sintering}

The crystallite size in modern ceramic powders is usually much less than $1 \mu \mathrm{m}$ and ranges down to tens of nanometers. While these crystallites have high surface area and 
Sampling and specimen preparation

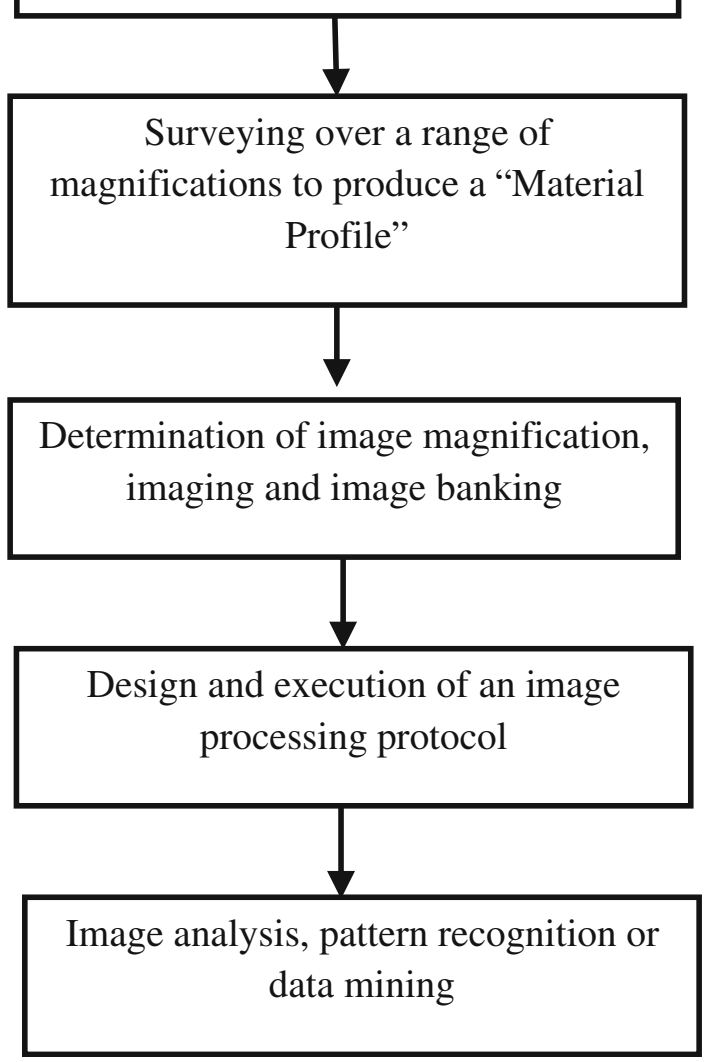

Fig. 1 The conceptual steps in microstructure mining

provide large driving force for sintering, they are often aggregated and result in more complex paths of microstructure evolution that detract from the potential of the powder to sinter at the lowest possible temperatures in the shortest periods of time. In the analysis of sintering kinetics, it is quite common to reduce the effect of the microstructure on sintering to two important parameters, the solid volume fraction and the grain size. In fact, the kinetic models of sintering allow the change in solid volume fraction to be modeled purely in terms of the change in grain size in isothermal sintering. This is a consequence of the assumed diffusional mechanisms by which vacancies migrate between pore boundaries (vacancy sources) and grain boundaries (vacancy sinks). Importantly, the limiting diffusion distance should be half the interpore spacing. Therefore, the exact relationship between sintering rate and grain size depends on the assumptions of the models. In the case of the combined stage sintering model [8] mentioned above, the proportionality between volume strain rate $\mathrm{d} \rho$ / $\rho \mathrm{d} t$, the grain size $\lambda_{\mathrm{g}}$ and the pore size $\lambda_{\mathrm{p}}$ for isothermal sintering by volume diffusion is:

$\mathrm{d} \rho / \rho \mathrm{d} t \propto \lambda_{\mathrm{p}} / \lambda_{\mathrm{g}}^{4}$
Since $\lambda_{\mathrm{p}}$ is assumed to be proportional to the grain size Eq. 1 reduces to:

$\mathrm{d} \rho / \rho \mathrm{d} t \propto 1 / \lambda_{\mathrm{g}}^{3}$

For grain boundary diffusion, there is no pore size dependence so the proportionality becomes:

$\mathrm{d} \rho / \rho \mathrm{d} t \propto 1 / \lambda_{\mathrm{g}}^{4}$

Figure 2 shows an example of a polished and thermally etched microstructural section of partially sintered zirconia ceramic derived from an aggregated nanocrystalline powder. The ceramic was processed from powders with an average particle size of approximately $0.3 \mu \mathrm{m}$ containing individual $30 \mathrm{~nm}$ crystals assembled into porous aggregates. It is immediately apparent that there are several ways in which this microstructure does not reflect the assumptions of the kinetic models of sintering. Unlike the geometries of the sintering models, there are many triple points that do not contain pores. Indeed dense aggregates containing many grains can be observed throughout the microstructure. Therefore the assumed equivalence of grain size and pore separation in the kinetic models of sintering do not appear to apply. While casual observation of the microstructure might lead to the conclusion that the model geometries of the kinetic models of sintering do not apply, it must be established if these models are statistically representative. Inspection of Eqs. 1-3 suggests that plots of log of volume strain rate against the log of grain size should yield a linear plot for which the slope represents the grain size exponent. The exponent must have a value of 3 for volume diffusion or a value of 4 for grain boundary diffusion, the expected sintering mechanisms. The volume strain rate in sintering can be determined from the change in solid volume fraction with time during isothermal sintering measured from

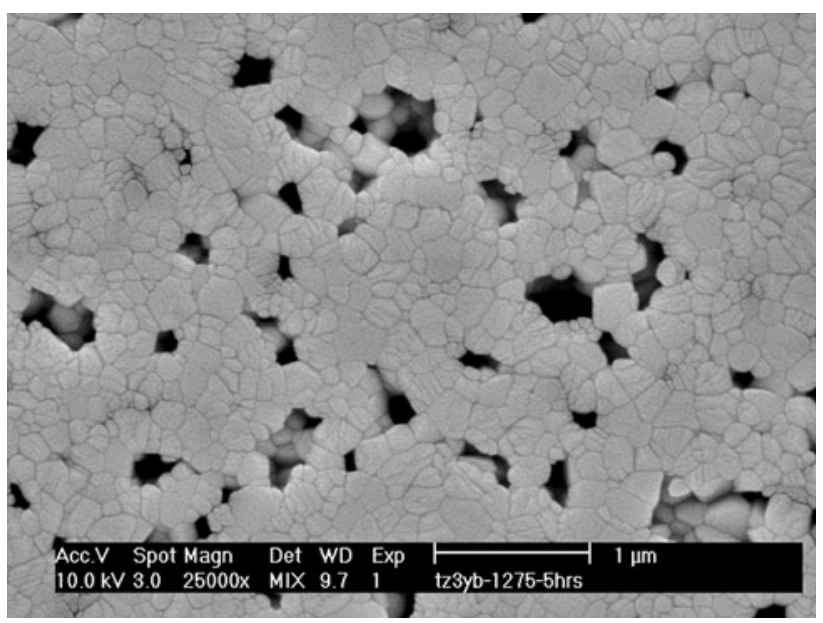

Fig. 2 A SEM micrograph of a partially sintered zirconia ceramic after $5 \mathrm{~h}$ at $1,275{ }^{\circ} \mathrm{C}$ 


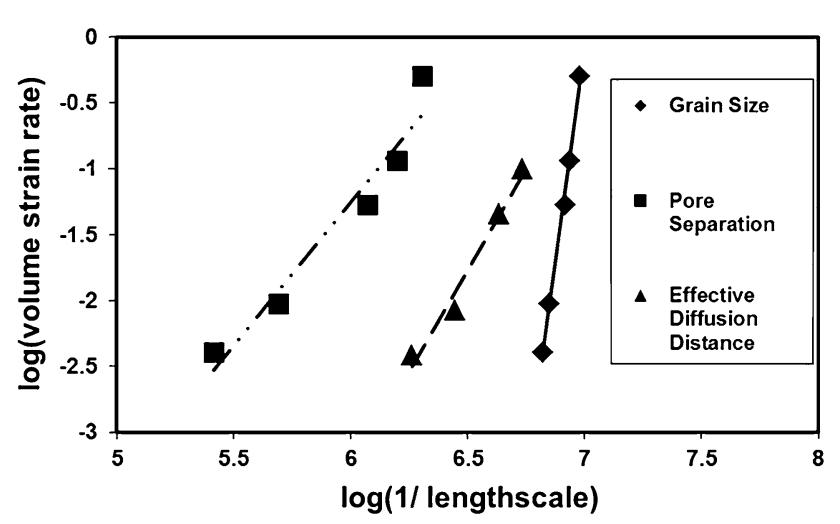

Fig. 3 Relationship between the volume strain rate and the measured lengthscales in the microstructure of zirconia sintered at $1,275{ }^{\circ} \mathrm{C}$

interrupted sintering experiments. The grain size can be determined from the grain intercept $\lambda_{\mathrm{g}}$ that is defined as follows:

$\lambda_{\mathrm{g}}=4 V_{\mathrm{s}} /\left(2 S_{\mathrm{v}}^{\mathrm{ss}}+S_{\mathrm{v}}^{\mathrm{sv}}\right)$,

where $V_{\mathrm{s}}$ is the solid volume fraction, $S_{\mathrm{v}}^{\mathrm{ss}}$ is the surface area density of grain boundaries, and $S_{\mathrm{v}}^{\mathrm{sv}}$ is the surface area density of pore boundaries. All of these quantities can be measured on the microstructural sections and the value of $V_{\mathrm{s}}$ from the microstructural measurement must agree with independent measurements using the Archimedes method if the images are to be considered representative. The consequent plot of the volume strain rate for isothermal sintering of zirconia at $1,275^{\circ} \mathrm{C}$ against grain size, shown in Fig. 3, gave a grain size exponent for sintering of 12 . Such a value is well above the values of 3 or 4 required by the expected sintering mechanisms. Importantly, the relationship suggests that the grain size is not increasing fast enough during isothermal sintering to account for the observed decrease in sintering rate. If the grain size does not correctly represent the diffusion distance that controls sintering, one might suspect that the average pore separation, might show a better correlation. The pore separation $\lambda_{\mathrm{ps}}$ can be easily defined from the same intercept data used to determine the grain intercept as follows:

$\lambda_{\mathrm{ps}}=4 V_{\mathrm{s}} / S_{\mathrm{v}}^{\mathrm{sv}}$

The plot shown in Fig. 3 gave a pore separation exponent close to 2. While such an exponent is closer to the expected values from the sintering models, it is low and the pore separation defined in Eq. 5 thereby overestimates the diffusion distance.

Using the microstructure mining methodology to observe these microstructures at a range of lengthscales, it was readily apparent that the pores were not randomly positioned in the microstructure but decorate the periphery of dense aggregates as shown in Fig. 2. Therefore, the pore separation measurements evaluated along randomly oriented lines using Eq. 5 will cut through dense aggregates in directions that would not represent diffusion distances that limit sintering kinetics. The real diffusion distance will be biased toward the distance between the most closely spaced pores because they will contribute most of the diffusional flux of ions. In order to address this circumstance, a new effective diffusion distance was defined to generalize the applicability of the traditional models of sintering [15]. At the core of this approach is a flux-weighted diffusion distance that preferentially fills more closely spaced pores along the periphery of the aggregates. A new image analysis measurement method was designed that uses binary images of polished microstructure sections that distinguishes the pore phase from the solid but does not require grain boundary reconstruction. In the first phase of the analysis, pore boundary distance maps and pore boundary tessellation were used to select pixels in the solid ceramic halfway between adjacent pore surfaces. The distance to the pore boundary of each of the selected pixels on the tessellation cell boundary was estimated using their gray levels from the distance map. This distance was then averaged for all the pixels on the boundary of each tessellation cell. The average was itself averaged over the thousands of tessellation cells on a group of representative images to arrive at the effective diffusion distance for the material microstructure. This distance was much larger than the grain size. When the effective diffusion length was plotted against volume strain rate for densification, shown in Fig. 3, the lengthscale exponent was 3, suggesting that the zirconia was densifying by volume diffusion.

In theory, the exponent on the effective diffusion distance could be used to identify a sintering mechanism based on the value of the measured exponent, however, it is unlikely that experimental results will give exact values of 3 or 4 and so ambiguity will always remain. More importantly, the determination of the effective diffusion distance can be used to examine the microstructural sensitivity of sintering irrespective of the state of aggregation in the microstructure. The effective diffusion distance allows the basic assumptions of the kinetic models of sintering to be adapted for the presence of aggregated microstructures without modeling a range of microstructural aggregation states that is presently not well characterized. The effective diffusion distance will allow predictions of the sintering times required to remove all the observed pore populations. For applications that require the removal of all residual porosity such as optical transparency [16], this will be an important prediction by which decisions could be made regarding extra processing steps to reduce the state of aggregation in the powder prior to forming and sintering [17].

Despite the apparent benefits of the effective diffusion distance, it is a measurement that is limited to images that 
represent the average microstructure. This requires the use of imaging magnifications in which individual pores can be correctly imaged while maintaining enough pores in the images to represent the average microstructure in the measurement. The suitability of the images can be determined by measuring the local solid fraction from the images and comparing the average with the solid volume fraction determined by an independent measurement using Archimedes principle. These values must be consistent for the images to be considered representative of the average, however, aggregation of nanocrystalline powders can occur over a range of lengthscales and so it is quite possible that the pores cannot be properly imaged at magnifications that contain enough material to be representative without a prohibitively large effort in image montaging. Figure 4 shows a plot of the local solid volume fraction determined by line fraction measurements on images taken using a magnification at which the pores can be imaged but the images do not contain enough pores to represent the average microstructure. This "local solid fraction" is plotted against the solid volume fraction measured on the whole specimen by the Archimedes principle. If the images were representative then the results would fall on the diagonal line in Fig. 4. This is not the case for the experimental results. At low values of solid volume fraction, the microscopy measurements and the values from the Archimedes method are relatively close, however, as sintering continues the smaller pores sampled by the microscopy measurements rapidly disappear and the solid fractional line length increases much faster than the solid volume fraction measured using the Archimedes method. This suggests that the information from the images is biased and some pores are not being counted either because they are too small to be imaged or because they are too large and widely spaced to be sampled. In principle, this could be rectified by methods such as systematic random sampling during images but the result can also lead to interesting interpretations. For example, in final stage sintering (above 0.92 solid volume fraction) the difference between the two solid fraction measurements diminishes once again. Indeed, observation at lower magnification showed that in final stage sintering most of the porosity was present as areas of widely spaced microstructural damage shown in Fig. 5. These features obviously create a sampling issue but more importantly should drastically affect sintering kinetics. The damage is thought to be due to spatial heterogeneities in the unsintered microstructure that sinter more slowly and cause differential sintering. Any predictions of sintering time based on the local solid volume fraction would underestimate the sintering time to full density and the sintering behavior of the damaged areas would have to be studied. Clearly, the sintering of aggregated ceramic powders must involve both kinetic studies

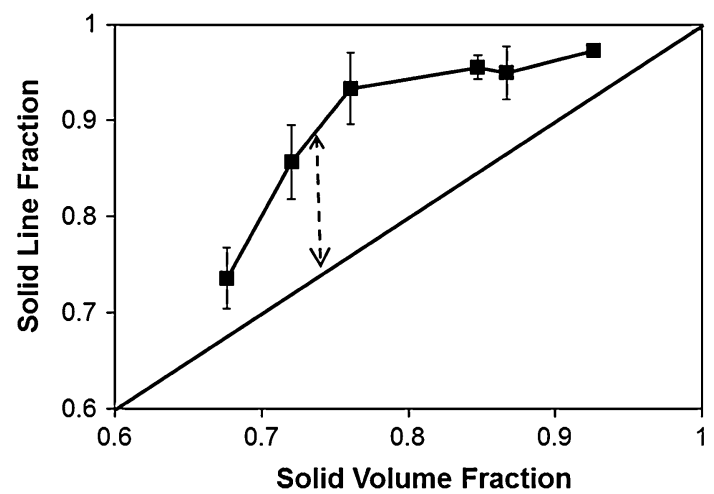

Fig. 4 Relationship between the solid volume fraction measured by the Archimedes principle and solid line fraction measured on SEM micrographs. The difference is thought to represent differential sintering

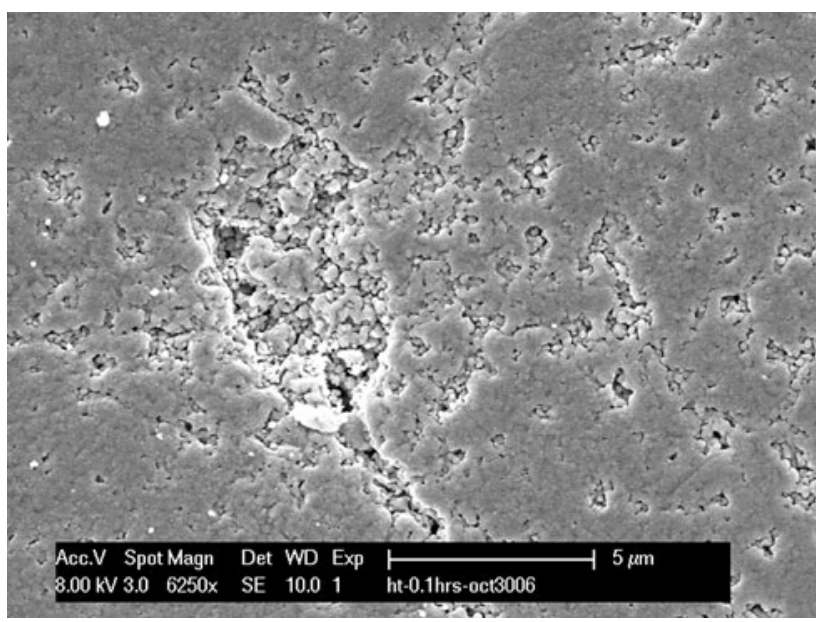

Fig. 5 An example of the large, widely spaced defects in partially sintered zirconia ceramics thought to be caused by differential sintering

and microstructural studies to clearly understand the effects of ceramics processing variables on properties such as transparency. Microstructure mining techniques combined with traditional models of sintering are just one such example of how this could be done.

\section{Summary}

Microstructure mining techniques can be used to study materials as complex systems. In particular, they could be used to define mesoscales associated with groups of microstructural features when they control material behavior. An example of a mesoscale flux-weighted effective diffusion distance was used to successfully study the sintering of aggregated nanocrystalline ceramic powders in the context of existing kinetic models of sintering. 
Acknowledgments The author would like to acknowledge colleagues: Richard McAfee, Tiandan Chen, Tom Hinklin, and Kevin Ewsuk for their contributions to these studies. The author also acknowledges the financial support of National Science Foundation under Grant 0900254.

\section{References}

1. K. Rajan, Materials informatics. Mater. Today 8, 38-45 (2005)

2. Integrated Computational Materials Engineering: A Transformational Discipline for Improved Competitiveness and National Security, Committee on Integrated Computational Materials Engineering, National Research Council published by National Academy Press (2008)

3. Materials Genome Initiative for Global Competiveness, National Science and Technology Council, Office of US President, June 2011

4. I.H. Witten, E. Frank, M.A. Hall, Data Mining: Practical Machine Learning Tools and Techniques, 3rd edn. (Elsevier Direct, Burlington, 2011)

5. O. Dengiz, A.E. Smith, I. Nettleship, Two-stage data mining for flaw identification in ceramic manufacture. Int. J. Prod. Res. 44, 2839-2851 (2006)

6. O. Dengiz, T. Chen, I. Nettleship, A.E. Smith, The effect of powder forming method on the pull-out flaw populations observed on polished surfaces of alumina ceramics. Mater. Sci. Eng. A A427, 160-166 (2006)

7. R.L. Coble, Sintering of crystalline solids I: intermediate and final stage diffusion models. J. Appl. Phys. 32, 787-792 (1961)
8. D. Hansen, R.P. Rusin, M.H. Teng, D.L. Johnson, Combined stage sintering model. J. Am. Ceram. Soc. 75, 1129-1135 (1992)

9. H.H. Su, D.L. Johnson, The master sintering curve: a practical approach to sintering. J. Am. Ceram. Soc. 79, 3211-3217 (1996)

10. R.T. DeHoff, The dynamics of microstructural change, in Treatise on Materials Science and Technology, vol. 1, ed. by $\mathrm{H}$. Herman (Academic Press, New York, 1972), pp. 247-292

11. C.A. Handwerker, R.M. Cannon, R.L. Coble, Final stage sintering of $\mathrm{MgO}$, in Structure and Properties of $\mathrm{MgO}$ and $\mathrm{Al}_{2} \mathrm{O}_{3}$, Advances in Ceramics, vol. 10, ed. by W.D. Kingery (American Ceramics Society, Columbus, 1984), pp. 619-643

12. M.P. Harmer, Use of solid-solution additives in ceramics processing, in Structure and Properties of $\mathrm{MgO}$ and $\mathrm{Al}_{2} \mathrm{O}_{3}$, Advances in Ceramics, vol. 10, ed. by W.D. Kingery (American Ceramics Society, Columbus, 1984), pp. 679-696

13. K.A. Berry, M.P. Harmer, Effect of $\mathrm{MgO}$ on microstructure development in $\mathrm{Al}_{2} \mathrm{O}_{3}$. J. Am. Ceram. Soc. 69, 143-149 (1986)

14. I. Nettleship, W.S. Slaughter, Dimensionless parameters of microstructure pathways during sintering. J. Am. Ceram. Soc. 81, 700-714 (1998)

15. T. Chen, I. Nettleship, R.J. McAfee, T.R. Hinklin, K.G. Ewsuk, An experimental measurement of effective diffusion distance for the sintering of ceramics. J. Am. Ceram. Soc. 92, 1481-1486 (2009)

16. A. Krell, P. Blank, H.W. Ma, T. Hutzler, M. Nebelung, Processing of high-density submicrometer $\mathrm{Al}_{2} \mathrm{O}_{3}$ for new applications. J. Am. Ceram. Soc. 86, 446-453 (2003)

17. A.J. Krell, J. Klimke, The effect of homogeneity of particle coordination on solid state sintering of transparent alumina. J. Am. Ceram. Soc. 89, 1985-1992 (2006) 